University of Mannheim / Department of Economics

Working Paper Series

\title{
Platform Competition: Who Benefits from Multihoming?
}

\author{
Paul Belleflamme Martin Peitz
}

Working Paper 17-05

November 2017 


\title{
Platform Competition: Who Benefits from Multihoming?*
}

\author{
Paul Belleflamme ${ }^{\dagger}$ \\ Martin Peitz \\ Aix-Marseille University \\ University of Mannheim
}

This version: November 2017

\begin{abstract}
Competition between two-sided platforms is shaped by the possibility of multihoming. If users on both sides singlehome, each platform provides users on either side exclusive access to its users on the other side. In contrast, if users on one side can multihome, platforms exert monopoly power on that side and compete on the singlehoming side. This paper explores the allocative effects of such a change from single- to multihoming. Our results challenge the conventional wisdom, according to which the possibility of multihoming hurts the side that can multihome, while benefiting the other side. This is not always true: the opposite may happen or both sides may benefit.
\end{abstract}

Keywords: Network effects, two-sided markets, platform competition, competitive bottleneck, multihoming

JEL-Classification: D43, L13, L86

\footnotetext{
${ }^{*}$ We thank Markus Reisinger and Julian Wright for helpful comments. Martin Peitz gratefully acknowledges financial support from the Deutsche Forschungsgemeinschaft (PE 813/2-2).

${ }^{\dagger}$ Aix-Marseille Univ., CNRS, EHESS, Centrale Marseille, AMSE; Paul.Belleflamme@univ-amu.fr. Other affiliations: KEDGE Business School and CESifo.

${ }^{\ddagger}$ Department of Economics and MaCCI, University of Mannheim, 68131 Mannheim, Germany, Martin.Peitz@gmail.com. Other affiliations: CEPR, CESifo, and ZEW.
} 


\section{Introduction}

Two-sided platforms cater to the tastes of two audiences - in many instances, buyers and sellers. Decisions among these audiences are interdependent because of positive cross-group external effects. One of the principle achievements of the literature on two-sided markets has been to characterize the price structure and associated price distortions in alternative market environments. Competing platforms have to take into account the effect of a price change to participation levels not only on the market side directly affected, but also indirect effects arising from altered participation on the other side.

A possible market environment is that both sides singlehome. To reach a particular agent on one side, an agent from the other side has to be on the same platform. If a platform lures an agent from either side away from a competitor onto its site, this platform becomes more attractive to agents on the other side, as more transaction partners become available on the platform's site and fewer partners are available on the competing site. Another possible market environment is that agents on one side can multihome and agents on the other can singlehome. This is the so-called competitive bottleneck, which has been described in these terms:

"Here, if it wishes to interact with an agent on the singlehoming side, the multihoming side has no choice but to deal with that agent's chosen platform. Thus, platforms have monopoly power over providing access to their singlehoming customers for the multihoming side. This monopoly power naturally leads to high prices being charged to the multihoming side, and there will be too few agents on this side being served from a social point of view [...]. By contrast, platforms do have to compete for the singlehoming agents, and high profits generated from the multihoming side are to a large extent passed on to the singlehoming side in the form of low prices (or even zero prices)." (Armstrong, 2006, pp. 669-670)

This insight has been appreciated and reproduced in various policy documents. For instance, in a recent report, the German Cartel Office writes: ${ }^{1}$

"Armstrong analyses a constellation which he describes as competitive bottlenecks with 'one side applying singlehoming, the other one multihoming'. In this scenario, the platforms were competing for users on the singlehoming side. Accordingly, on the multihoming side, platforms provided monopolistic access to singlehoming users who were members of the platform. Regarding the framework of the model reviewed, this led to a monopolistic price on the multihoming side, while the price on the singlehoming side would be fairly low as a result of platforms competing for users on this side. In this respect, this may result in an inefficient price structure despite potentially intensive platform competition (on the singlehoming side)." (BKartA, 2016, p. 58)

\footnotetext{
${ }^{1}$ Another instance is the statement by the European Commission in OECD (2009, p. 169).
} 
In this paper, we take a closer look at the price and surplus effects of multihoming. We compare the competitive bottleneck to the two-sided singlehoming market environment. In the latter, platforms compete on both sides of the market, whereas on the former they compete on only one. One may therefore be tempted to conclude that an audience that obtains the possibility to multihome faces higher prices and obtains a lower surplus, while the other audience faces lower prices and obtains a higher surplus. Also, since in the competitive bottleneck, platforms compete on only one side, one may expect that their profits are higher than in the market environment in which both audiences singlehome. Yet, the effect of making one side multihome instead of singlehome is less straightforward than what may in general be perceived. While it is true that platforms exert monopoly power over the multihoming side, participants on this side may actually benefit from multihoming.

As Evans and Schmalensee (2012, p. 16) observe, ${ }^{2}$ "in software platforms, for instance, the price structure appears to be the opposite of what the competitive bottlenecks theory would predict. Most personal computer users rely on a single software platform, while most developers write for multiple platforms. Yet personal computer software providers generally make their platforms available for free, or at low cost, to applications developers and earn profits from the single-homing user side." As our analysis will reveal, while this observation runs counter the claim that the multihoming side faces high prices, it is perfectly compatible with the competitive bottleneck model.

For the sake of concreteness, we assume that the seller side is the side of the market which potentially can multihome, while buyers always singlehome. Our main findings are as follows. When going from singlehoming to multihoming on one side, prices on both sides of the market always move in opposite directions. It is not necessarily the case that sellers pay a higher fee and buyers, a lower fee; the opposite may occur because sellers may pay a low price to start with in the competitive bottleneck case. Platforms prefer to impose exclusivity to sellers (i.e., to prevent them from multihoming) if the sellers' intrinsic value (the difference between their stand-alone benefit and the marginal cost of accommodating them) is not too large. There exist configurations of parameters for which this condition is always, or never, satisfied. Buyers tend to prefer the competitive bottleneck environment when they value a lot the presence of sellers and sellers find it profitable to multihome; they are then more likely to interact with a larger set of buyers and to be charged lower fees. However, it may also happen that platforms charge higher fees to buyers when sellers multihome than when they singlehome, and that this negative price effect outweighs the positive participation effect, leading buyers to prefer the two-sided singlehoming environment. As for sellers, if they perceive the two platforms as more differentiated and if they exert weaker cross-group effects on buyers, then they are more likely to be better off in the competitive bottleneck case.

Combining these findings, we obtain three important insights about how the surplus effects play out for the three groups. First, the resulting market outcome may have the feature that buyers, sellers and platforms are all better off when sellers are allowed to multihome. Second,

\footnotetext{
${ }^{2}$ Evans, Hagiu, and Schmalensee (2006) made the same point.
} 
whenever platforms benefit from imposing exclusivity, they necessarily hurt buyers and possibly also sellers. Thus, in an environment with potential seller multihoming, an agency should prohibit the use of exclusivity of the seller side if its aim is to maximize buyer surplus. Third, whenever buyers suffer from seller multihoming, platforms and sellers benefit from it.

While comparing a two-sided singlehoming model to a competitive bottleneck model is an interesting exercise, it may appear to be of little practical relevance because singlehoming on both sides is observed in only few market environments. We want to challenge this view. First, while multihoming may be feasible for some users on one side, it may well be the case that due to habits or other latent factors a fraction of users does not consider the possibility of multihoming. Then, a comparison between two-sided multihoming and the competitive bottleneck is an extreme version of a comparison between markets in which such habits and other latent factor are present and those in which they are not. Second, the present comparison informs policy makers about possible effects when taking actions to enable multihoming or prohibiting exclusive dealing for a fraction of sellers (or, in the flip side of the model, of buyers). The former may take the form of aggregators combining the functionalities and listings by both platforms.

There exists surprisingly little work that studies the competitive effects of multihoming, despite the policy debate about means to encourage multihoming. In the seminal paper by Armstrong (2006) both market environments - that is, two-sided single-homing and competitive bottleneck - are analyzed in detail, but no comparison is undertaken. We follow his approach of considering platforms that are horizontally differentiated on both sides of the market and charge access fees to each side.

As an alternative, platforms may charge transaction fees, as analyzed in Rochet and Tirole (2003). For an insightful discussion of the use of different price instruments, see Rochet and Tirole (2006). While it would be interesting, to extend our analysis to other price instruments, we restrict attention to access fees, which is the natural assumption to make when platforms cannot monitor transactions.

Armstrong and Wright (2007) endogenize the multihoming decision of buyers and sellers. In their setting, the competitive bottleneck model emerges endogenously as one side decides not to multihome along the equilibrium path. They, as well as the rest of the literature, do not look at the surplus effects of the possibility of multihoming. In general, there exists little work on surplus effects in markets with two-sided platforms. An exception is Anderson and Peitz (2017) - they evaluate surplus effects of policy interventions in the competitive bottleneck world.

The remainder of the paper is organized as follows. We first lay out the model (Section 2) and solve it when both sides singlehome (Section 3) and when one side is allowed to multihome (Section 4). We are then in a position to derive our main results by comparing the two settings (Section 5). After showing that our results are robust to a more general formulation of the utility of multihomers (Section 6), we propose some concluding remarks (Section 7). 


\section{The model}

Two platforms compete to facilitate the interaction between a unit mass of sellers and a unit mass of buyers, with this interaction generating positive cross-group external effects. Following Armstrong (2006), we assume that platforms compete in membership fees and that buyers and sellers perceive them as horizontally differentiated. Horizontal differentiation is modeled in the Hotelling fashion: platforms are located at the extreme points of the unit interval and face constant $\operatorname{costs} c_{s}$ and $c_{b}$ for each additional seller and buyer, respectively; sellers and buyers are uniformly distributed on this unit interval and incur an opportunity cost of visiting a platform that increases linearly in distance at rates $\tau_{s}$ and $\tau_{b}$, respectively.

We assume the following form of interaction between buyers and sellers on a platform: buyers purchase one unit of the perfectly differentiated product offered by each seller who is active on the platform; each trade generates a benefit $b_{b}$ for the buyer and a profit $b_{s}$ for the seller. ${ }^{3}$ Buyers and sellers also derive a stand-alone benefit from visiting a platform; we assume that these benefits are equal across platforms and we note them $r_{b}$ and $r_{s}$. Letting $n_{b}^{i}$ and $n_{s}^{i}$ denote the mass of buyers and sellers active on platform $i$, and noting $m_{b}^{i}$ and $m_{s}^{i}$ the membership fees that platform $i$ charges buyers and sellers, we can express buyer and seller surpluses of visiting platform $i$ (gross of any opportunity cost) as

$$
v_{s}^{i}=r_{s}+n_{b}^{i} b_{s}-m_{s}^{i} \quad \text { and } \quad v_{b}^{i}=r_{b}+n_{s}^{i} b_{b}-m_{b}^{i} .
$$

We consider a two-stage game in which platforms $i \in\{1,2\}$ set membership fees on each side of the market, $m_{s}^{i}, m_{b}^{i}$, simultaneously in stage 1 and buyers and sellers simultaneously make their subscription decisions in stage 2 . We compare different settings for stage 2 according to whether participants in one or the other group choose at most one platform (i.e., to 'singlehome') or also have the option to be active on both platforms (i.e., to 'multihome'). We solve for subgameperfect equilibria and will restrict attention to parameter constellations such that both platforms will be active in equilibrium (we will provide precise assumptions below).

As a benchmark, consider the case that cross-group external effects are zero-i.e., $b_{b}=b_{s}=0$. Thus, if participants on a particular side are singlehoming, we solve the standard Hotelling model and obtain $c_{g}+\tau_{g}$ as equilibrium price on side $g \in\{b, s\}$, provided that there is full market coverage. This requires that $r_{g}-\tau_{g} / 2-\left(c_{g}+\tau_{g}\right)>0$ or, equivalently, $r_{g}-c_{g}>(3 / 2) \tau_{g}$ holds. If participants are multihoming, their choice of buying from one platform is independent of the pricing of the other platform, and each platform solves a monopoly problem. Solving the first-order condition of profit maximization when the marginal consumer is in the interior of the [0,1]-interval, we obtain the price $\left(r_{g}+c_{g}\right) / 2$. At this price, the marginal consumer is indeed in the interior if $r_{g}-\tau_{g}-\left(r_{g}+c_{g}\right) / 2<0$, which is equivalent to $r_{g}-c_{g}<2 \tau_{g}$. In this case, the price under multihoming is less than under singlehoming if and only if $\left(r_{g}+c_{g}\right) / 2<c_{g}+\tau_{g}$ or, equivalently, $r_{g}-c_{g}<2 \tau_{g}$. To summarize, for $(3 / 2) \tau_{g}<r_{g}-c_{g}<2 \tau_{g}$, the price under

\footnotetext{
${ }^{3}$ It is assumed, quite realistically, that in a seller-buyer relationship, prices or terms of transaction are independent of the membership fee that applies to buyers and sellers.
} 
multihoming - which coincides with the monopoly price in a single-product monopoly problemis less than the price under singlehoming - which coincides with the standard Hotelling duopoly problem. The reason for this counterintuitive result is that the firm faces a more elastic demand when the consumers' outside option is a constant rather than the competitor's offer (which becomes increasingly attractive when the firm wants to reach consumers located further away) that is, the same price cut leads to a larger increase in the demand in the monopoly than in the duopoly setting.

\section{Two-sided singlehoming}

In this section we consider a market environment in which both sides of the market singlehome. Our aim is to characterize the equilibrium in which both platforms are active. ${ }^{4}$

If buyers and sellers singlehome, the seller and the buyer who are indifferent between the two platforms are respectively located at $x_{s}$ and $x_{b}$ such that $v_{s}^{1}-\tau_{s} x_{s}=v_{s}^{2}-\tau_{s}\left(1-x_{s}\right)$ and $v_{b}^{1}-\tau_{b} x_{b}=v_{b}^{2}-\tau_{b}\left(1-x_{b}\right)$. It follows that $n_{s}^{1}=x_{s}, n_{s}^{2}=1-x_{s}, n_{b}^{1}=x_{b}$, and $n_{b}^{2}=1-x_{b}$ and the total number of each side's agents on the two platforms adds up to 1: $n_{s}^{1}+n_{s}^{2}=n_{b}^{1}+n_{b}^{2}=1$. Combining the indifference equations together with the expressions of $v_{s}^{i}$ and $v_{b}^{i}$, we obtain the following expressions for the numbers of buyers and sellers at the two platforms:

$$
\left\{\begin{array}{l}
n_{s}^{i}\left(n_{b}^{i}\right)=\frac{1}{2}+\frac{1}{2 \tau_{s}}\left(\left(2 n_{b}^{i}-1\right) b_{s}-\left(m_{s}^{i}-m_{s}^{j}\right)\right), \\
n_{b}^{i}\left(n_{s}^{i}\right)=\frac{1}{2}+\frac{1}{2 \tau_{b}}\left(\left(2 n_{s}^{i}-1\right) b_{b}-\left(m_{b}^{i}-m_{b}^{j}\right)\right) .
\end{array}\right.
$$

Solving this linear equation system, we derive the equilibrium number of buyers and sellers at stage 2 as a function of the membership fees:

$$
\begin{aligned}
& n_{s}^{i}\left(m_{s}^{i}, m_{s}^{j}, m_{b}^{i}, m_{b}^{j}\right)=\frac{1}{2}+\frac{b_{s}\left(m_{b}^{j}-m_{b}^{i}\right)+\tau_{b}\left(m_{s}^{j}-m_{s}^{i}\right)}{2\left(\tau_{b} \tau_{s}-b_{b} b_{s}\right)}, \\
& n_{b}^{i}\left(m_{s}^{i}, m_{s}^{j}, m_{b}^{i}, m_{b}^{j}\right)=\frac{1}{2}+\frac{b_{b}\left(m_{s}^{j}-m_{s}^{i}\right)+\tau_{s}\left(m_{b}^{j}-m_{b}^{i}\right)}{2\left(\tau_{b} \tau_{s}-b_{b} b_{s}\right)} .
\end{aligned}
$$

Platform $i$ chooses $m_{s}^{i}$ and $m_{b}^{i}$ to maximize $\Pi^{i}=\left(m_{s}^{i}-c_{s}\right) n_{s}^{i}(\cdot)+\left(m_{b}^{i}-c_{b}\right) n_{b}^{i}(\cdot)$. At the symmetric equilibrium $\left(m_{s}^{1}=m_{s}^{2} \equiv m_{s}\right.$ and $\left.m_{b}^{1}=m_{b}^{2} \equiv m_{b}\right)$, the first-order conditions can be written as

$$
\left\{\begin{array}{l}
m_{s}=c_{s}+\tau_{s}-\frac{b_{b}}{\tau_{b}}\left(b_{s}+m_{b}-c_{b}\right), \\
m_{b}=c_{b}+\tau_{b}-\frac{b_{s}}{\tau_{s}}\left(b_{b}+m_{s}-c_{s}\right) .
\end{array}\right.
$$

The equilibrium membership fee for the sellers is equal to marginal costs plus the productdifferentiation term as in the standard Hotelling model, adjusted downward by the term $\frac{b_{b}}{\tau_{b}}\left(b_{s}+\right.$ $\left.m_{b}-c_{b}\right)$. As pointed out by Armstrong (2006), to understand this term, note from expression (1) that each additional seller attracts $b_{b} / \tau_{b}$ additional buyers. These additional buyers allow the intermediary to extract $b_{s}$ per seller without affecting the sellers' surplus. In addition, each of

\footnotetext{
${ }^{4}$ Our analysis follows Armstrong (2006). A textbook treatment can be found in Belleflamme and Peitz (2015).
} 
the additional $b_{b} / \tau_{b}$ buyers generates a margin of $m_{b}-c_{b}$ to the platform. Thus $\frac{b_{b}}{\tau_{b}}\left(b_{s}+m_{b}-c_{b}\right)$ represents the value of an additional buyer to the platform. The same holds on the buyers' side.

Solving the system of first-order conditions (with full participation) gives explicit expressions for equilibrium membership fees (where the superscript $2 S$ denotes 'two-sided singlehoming'):

$$
m_{s}^{2 S}=c_{s}+\tau_{s}-b_{b} \text { and } m_{b}^{2 S}=c_{b}+\tau_{b}-b_{s} .
$$

We observe that the equilibrium membership fee for one group is equal to the usual Hotelling formulation (marginal cost plus transportation cost) adjusted downward by the cross-group external effect that this group exerts on the other group (see Armstrong, 2006).

As platforms set the same fees at equilibrium, the indifferent participants of both sides are located at $1 / 2$, meaning that $n_{s}^{2 S}=n_{b}^{2 S}=1 / 2$. It follows that in equilibrium, a seller and a buyer obtain, respectively, a surplus (gross of their transport cost) given by

$$
v_{s}^{2 S}=h_{s}+\frac{1}{2} b_{s}-\tau_{s}+b_{b} \text { and } v_{b}^{2 S}=h_{b}+\frac{1}{2} b_{b}-\tau_{b}+b_{s},
$$

where $h_{g} \equiv r_{g}-c_{g}$ denotes the difference between the stand-alone benefit and the cost of accommodating a participant of side $g \in\{b, s\}$. Thus sellers' and buyers' aggregate surpluses are calculated respectively as

$$
\begin{aligned}
& P S^{2 S}=v_{s}^{2 S}-2 \int_{0}^{\frac{1}{2}} \tau_{s} x d x=h_{s}-\frac{5}{4} \tau_{s}+\frac{1}{2} b_{s}+b_{b}, \\
& C S^{2 S}=v_{b}^{2 S}-2 \int_{0}^{\frac{1}{2}} \tau_{b} x d x=h_{b}-\frac{5}{4} \tau_{b}+\frac{1}{2} b_{b}+b_{s} .
\end{aligned}
$$

Finally, equilibrium profits are the same for both platforms and are computed as

$$
\Pi^{2 S}=\frac{1}{2}\left(\tau_{b}+\tau_{s}-b_{b}-b_{s}\right) .
$$

They are increasing in the degree of product differentiation on both sides of the market (as in the Hotelling model) and decreasing in the buyers' and sellers' surplus for each transaction, i.e., the magnitude of the cross-group external effects. The intuition for the latter result is the following: as cross-group external effects increase, platforms compete more fiercely to attract additional agents on each side as they become more valuable.

A series of conditions have to be met for the previous equilibrium to be valid. First, the second-order conditions of the profit-maximization program are $\tau_{b} \tau_{s}>b_{b} b_{s}$ and $4 \tau_{b} \tau_{s}>\left(b_{b}+\right.$ $\left.b_{s}\right)^{2}$. Both conditions require that the transportation cost parameters $\tau_{b}$ and $\tau_{s}$ (which measure the horizontal differentiation between the two platforms) are sufficiently large with respect to the gains from trade $b_{b}$ and $b_{s}$ (which measure the cross-group external effects). These conditions are also sufficient to have a unique and stable equilibrium in which both platforms are active. We check that the former condition makes sure that the number of members of one group at one platform, $n_{s}^{i}(\cdot)$ or $n_{b}^{i}(\cdot)$, decreases not only with the membership fee that they have to pay but also with the membership fee that the other group has to pay on this platform. ${ }^{5}$ We also

\footnotetext{
${ }^{5}$ For stronger cross-group external effects and/or weaker horizontal differentiation (i.e., for $b_{b} b_{s}>\tau_{b} \tau_{s}$ ), the number of agents on one platform would be an increasing function of their membership fee and the market would tip; i.e., all buyers and sellers would choose the same platform.
} 
observe that the latter condition is more restrictive than the former. Furthermore, we need to guarantee full participation on the two sides; that is, the indifferent participant on each side (located at 1/2) must have a positive net surplus at equilibrium: on the seller side, $v_{s}^{2 S}-\frac{1}{2} \tau_{s}>0$ or $2 h_{s}>3 \tau_{s}-b_{s}-2 b_{b}$; on the buyer side $v_{b}^{2 S}-\frac{1}{2} \tau_{b}>0$ or $2 h_{b}>3 \tau_{b}-b_{b}-2 b_{s}$. In sum, we make the following set of assumptions for the two-sided singlehoming case.

Assumption 1 In the two-sided singlehoming case, parameters satisfy

$$
\begin{aligned}
& 4 \tau_{b} \tau_{s}>\left(b_{b}+b_{s}\right)^{2} \\
& 2 h_{s}>3 \tau_{s}-b_{s}-2 b_{b} \\
& 2 h_{b}>3 \tau_{b}-b_{b}-2 b_{s}
\end{aligned}
$$

\section{Multihoming on one side (competitive bottlenecks)}

Suppose now that sellers have the possibility to multihome (i.e., to be active on both platforms at the same time), while buyers continue to singlehome. We assume that the decision whether to subscribe to one platform is independent of the decision whether to subscribe to the other platform. In particular, if a seller $x$ is subscribed to both platforms his surplus is $r_{s}+n_{b}^{1} b_{s}$ $m_{s}^{1}-\tau_{s} x+r_{s}+n_{b}^{2} b_{s}-m_{s}^{2}-\tau_{s}(1-x)=2 r_{s}+b_{s}-\tau_{s}-\left(m_{s}^{1}+m_{s}^{2}\right)$, which is independent of his location. According to our assumption, a multihoming seller enjoys the stand-alone benefit on both platforms - i.e., platforms are symmetric but provide different services leading to standalone benefits that can be combined when multihoming. ${ }^{6}$

Sellers can be divided into three subintervals on the unit interval: those sellers located "on the left" register with platform 1 only, those located "around the middle" register with both platforms, and those located "on the right" register with platform 2 only. At the boundaries between these intervals, $x_{i 0}$, we find the sellers who are indifferent between visiting platform $i$ $(i \in\{1,2\})$ and not visiting this platform. Their locations are found as, respectively, $x_{10}$ such that $r_{s}+n_{b}^{1} b_{s}-m_{s}^{1}=\tau_{s} x_{10}$, and $x_{20}$ such that $r_{s}+n_{b}^{2} b_{s}-m_{s}^{2}=\tau_{s}\left(1-x_{20}\right)$. We assume for now that $0<x_{20}<x_{10}<1$ (we provide necessary and sufficient conditions below), so that $n_{s}^{1}=x_{10}$ and $n_{s}^{2}=1-x_{20}$, with the multihoming sellers being located between $x_{20}$ and $x_{10}$. As far as buyers are concerned, we have the same situation as in the previous section. The number of buyers and sellers visiting each platform are thus respectively given by

$$
n_{b}^{i}=\frac{1}{2}+\frac{b_{b}\left(n_{s}^{i}-n_{s}^{j}\right)-\left(m_{b}^{i}-m_{b}^{j}\right)}{2 \tau_{b}} \text { and } n_{s}^{i}=\frac{r_{s}+n_{b}^{i} b_{s}-m_{s}^{i}}{\tau_{s}} .
$$

Solving this system of four equations in four unknowns, we obtain buyers' and sellers' participation as a function of subscription fees and the parameters of the model,

$$
\left\{\begin{array}{l}
n_{b}^{i}=\frac{1}{2}+\frac{b_{b}\left(m_{s}^{j}-m_{s}^{i}\right)+\tau_{s}\left(m_{b}^{j}-m_{b}^{i}\right)}{2\left(\tau_{b} \tau_{s}-b_{b} b_{s}\right)} \\
n_{s}^{i}=\frac{b_{s}}{\tau_{s}}\left(\frac{1}{2}+\frac{b_{b}\left(m_{s}^{j}-m_{s}^{i}\right)+\tau_{s}\left(m_{b}^{j}-m_{b}^{i}\right)}{2\left(\tau_{b} \tau_{s}-b_{b} b_{s}\right)}\right)+\frac{r_{s}-m_{s}^{i}}{\tau_{s}} .
\end{array}\right.
$$

\footnotetext{
${ }^{6}$ This assumption differs form Armstrong and Wright (2007) who assume that the services that give rise to the stand-alone utility are the same on both platforms. We discuss the implications of our assumption in Section 6 .
} 
The maximization problems of the two platforms are the same as above. Platform 1's best responses are implicitly defined by the first-order conditions, which can be expressed as

$$
\begin{aligned}
& m_{b}^{1}=\frac{-\left(b_{b}+b_{s}\right) m_{s}^{1}+b_{b} m_{s}^{2}+\tau_{s} m_{b}^{2}-b_{s}\left(b_{b}-c_{s}\right)+\tau_{s}\left(\tau_{b}+c_{b}\right)}{2 \tau_{s}}, \\
& m_{s}^{1}=\frac{-\left(b_{b}+b_{s}\right) \tau_{s} m_{b}^{1}+b_{b} b_{s} m_{s}^{2}+b_{s} \tau_{s} m_{b}^{2}-b_{s} b_{b}\left(b_{s}+c_{s}+2 r_{s}\right)+b_{b} \tau_{s} c_{b}+\left(b_{s}+2 c_{s}+2 r_{s}\right) \tau_{b} \tau_{s}}{2\left(2 \tau_{b} \tau_{s}-b_{b} b_{s}\right)} .
\end{aligned}
$$

Solving the previous system of equations, we find the equilibrium membership fees, which are equivalent for both platforms (with the superscript $C B$ standing for 'competitive bottleneck'):

$$
\begin{aligned}
& m_{s}^{C B}=\frac{1}{2}\left(r_{s}+c_{s}\right)+\frac{1}{4}\left(b_{s}-b_{b}\right), \\
& m_{b}^{C B}=c_{b}+\tau_{b}-\frac{b_{s}}{4 \tau_{s}}\left(b_{s}+3 b_{b}+2 r_{s}-2 c_{s}\right) .
\end{aligned}
$$

On the seller side, platforms have monopoly power. If the platform focused only on sellers, it would charge a monopoly price equal to $\left(r_{s}+c_{s}\right) / 2+b_{s} / 4$ (assuming that each seller would have access to half of the buyers and, therefore, would have a gross willingness to pay equal to $b_{s} / 2$ ). We observe that this price is adjusted downward by $b_{b} / 4$ when the cross-group effect that sellers exert on the buyer side is taken into account. Similarly, on the buyer side, platforms charge the Hotelling price, $c_{b}+\tau_{b}$, less a term that depends on the size of the cross-group effects and on the parameters characterizing the seller side $\left(r_{s}, c_{s}\right.$, and $\left.\tau_{s}\right)$.

It is useful to compare price changes in the competitive bottleneck model to those in the twosided singlehoming model. We observe that the equilibrium membership fee for sellers is increasing in the strength of the cross-group effect in the competitive bottleneck model $\left(\partial m_{s}^{C B} / \partial b_{s}>0\right)$, whereas it is constant in the two-sided singlehoming model. This is due to the monopoly pricing feature on the multihoming side. Everything else equal, if sellers are multihoming, the platform operators directly appropriate part of the rent generated on the multihoming side by setting higher membership fees. This is not the case in the singlehoming world, where the membership fee does not react to the strength of the network effect on the same side since platforms compete for sellers (and buyers).

At equilibrium, seller and buyer participation is

$$
n_{b}^{C B}=\frac{1}{2} \text { and } n_{s}^{C B}=\frac{1}{4 \tau_{s}}\left(b_{b}+b_{s}+2 h_{s}\right) .
$$

This allows us to compute the equilibrium net surplus of sellers and buyers (gross of transportation cost and for one platform) as:

$$
\begin{aligned}
v_{s}^{C B} & =\frac{1}{4}\left(b_{b}+b_{s}+2 h_{s}\right), \\
v_{b}^{C B} & =\frac{1}{4 \tau_{s}}\left(b_{b}^{2}+4 b_{s} b_{b}+b_{s}^{2}+2\left(b_{b}+b_{s}\right) h_{s}\right)+h_{b}-\tau_{b} .
\end{aligned}
$$

Note that $v_{s}^{C B}$ is the per-platform seller's surplus (gross of transport costs). ${ }^{7}$ We observe that $v_{s}^{C B}$ and $v_{b}^{C B}$ are increasing in the net gain of the other side and in the net gain of the own side.

\footnotetext{
${ }^{7}$ Sellers located between $1-n_{s}^{C B}$ and $n_{s}^{C B}$ multihome and, therefore, earn a surplus of $2 v_{s}^{C B}$. On the other hand, $v_{s}^{C B}$ is the surplus earned by the sellers located between 0 and $1-n_{s}^{C B}$, who choose to visit platform 1 only, and by the sellers located between $n_{s}^{C B}$ and 1 , who choose to visit platform 2 only.
} 
Aggregated over all buyers, in equilibrium, buyers' surplus is

$$
C S^{C B}=v_{b}^{C B}-2 \int_{0}^{1 / 2} \tau_{b} x d x=\frac{1}{4 \tau_{s}}\left(\left(b_{b}+b_{s}\right)\left(b_{b}+b_{s}+2 h_{s}\right)+2 b_{b} b_{s}\right)+h_{b}-\frac{5}{4} \tau_{b} .
$$

Aggregated over all sellers, in equilibrium, sellers' surplus is computed as (using the definitions of $n_{s}^{C B}$ and $\left.v_{s}^{C B}\right)$ :

$$
\begin{aligned}
P S^{C B} & =\int_{0}^{1-n_{s}^{C B}}\left(v_{s}^{C B}-\tau_{s} x\right) d x+\int_{1-n_{s}^{C B}}^{n_{s}^{C B}}\left(2 v_{s}^{C B}-\tau_{s}\right) d x+\int_{n_{s}^{C B}}^{1}\left(v_{s}^{C B}-\tau_{s}(1-x)\right) d x \\
& =\frac{1}{\tau_{s}}\left(v_{s}^{C B}\right)^{2}=\frac{1}{16 \tau_{s}}\left(b_{b}+b_{s}+2 h_{s}\right)^{2} .
\end{aligned}
$$

We observe that the aggregated seller surplus is decreasing in the degree of platform differentiation on the seller side, increasing in the stand-alone benefit on the seller side, and increasing in cross-group external effects on both sides.

The platforms' equilibrium profits are

$$
\Pi^{C B}=\frac{1}{16 \tau_{s}}\left(8 \tau_{b} \tau_{s}-\left(b_{b}+b_{s}\right)^{2}-4 b_{b} b_{s}+4 h_{s}^{2}\right) .
$$

As above, a set of conditions need to be satisfied for this equilibrium to hold. The secondorder conditions are here $\tau_{b} \tau_{s}>b_{b} b_{s}$ and $8 \tau_{b} \tau_{s}>\left(b_{b}+b_{s}\right)^{2}+4 b_{b} b_{s}$, the second condition being more stringent than the first. Note that the latter condition implies that even if platforms do not offer stand-alone utilities $\left(h_{s}=r_{s}-c_{s}=0\right)$, equilibrium profits are strictly positive. These conditions also guarantee that a unique and stable equilibrium exists in which both platforms share the market - in short, sharing equilibrium. We also impose that some (but not all) sellers multihome at equilibrium (if some sellers multihome, this also implies that all sellers participate). This is the case if $1 / 2<n_{s}^{C B}<1$, which is equivalent to $2 \tau_{s}<b_{s}+b_{b}+2 h_{s}<4 \tau_{s}$. Finally, all buyers must be willing to participate; i.e., $r_{b}+n_{s}^{C B} b_{b}-m_{b}^{C B}-\frac{1}{2} \tau_{b}>0$ which is equivalent to $4 \tau_{s} h_{b}+2\left(b_{b}+b_{s}\right) h_{s}>6\left(\tau_{s} \tau_{b}-b_{s} b_{b}\right)-\left(b_{b}-b_{s}\right)^{2}$. We collect all these conditions in the following assumption.

Assumption 2 In the competitive bottleneck case (with multihoming sellers), parameters satisfy

$$
\begin{aligned}
& 8 \tau_{b} \tau_{s}>\left(b_{b}+b_{s}\right)^{2}+4 b_{b} b_{s} \\
& 2 h_{s}>2 \tau_{s}-b_{s}-b_{b} \\
& 2 h_{s}<4 \tau_{s}-b_{s}-b_{b} \\
& 4 \tau_{s} h_{b}+2\left(b_{b}+b_{s}\right) h_{s}>6\left(\tau_{s} \tau_{b}-b_{s} b_{b}\right)-\left(b_{b}-b_{s}\right)^{2}
\end{aligned}
$$

Comparing conditions in Assumptions 1 and 2, we note the following. First, the secondorder conditions are less demanding in the competitive bottleneck case than in the two-sided singlehoming case; that is, if (Soc2S) is satisfied, so is (SocCB). Second, for $\tau_{s}>b_{b}$, full participation of sellers is guaranteed in the competitive bottleneck case if full participation is guaranteed in the two-sided singlehoming case; that is, if (FPs2S) is met, then so is (FPsCB). 


\section{$5 \quad$ Singlehoming vs. multihoming}

In this section, we compare the sharing equilibrium of the two previous environments. Thus, the sets of assumptions 1 and 2 have to hold. Regrouping them, we impose: ${ }^{8}$

$$
\left\{\begin{array}{l}
4 \tau_{b} \tau_{s}>\left(b_{b}+b_{s}\right)^{2} \\
h_{b}>\max \left\{\frac{1}{2}\left(3 \tau_{b}-b_{b}-2 b_{s}\right), \frac{1}{4 \tau_{s}}\left(6\left(\tau_{s} \tau_{b}-b_{s} b_{b}\right)-\left(b_{b}-b_{s}\right)^{2}-2\left(b_{b}+b_{s}\right) h_{s}\right)\right\} \\
h_{s}^{\min } \equiv \max \left\{\frac{1}{2}\left(2 \tau_{s}-b_{s}-b_{b}\right), \frac{1}{2}\left(3 \tau_{s}-b_{s}-2 b_{b}\right), 0\right\}<h_{s}<h_{s}^{\max } \equiv \frac{1}{2}\left(4 \tau_{s}-b_{s}-b_{b}\right)
\end{array}\right.
$$

To start with, we ask when a sharing equilibrium can be supported in the two environments. The relevant assumption regarding the relationship between platform differentiation and cross-group external effects is (Soc2S) with two-sided singlehoming market and (SocCB) in the competitive bottleneck market. As mentioned above, the former implies the latter. This confirms the claims that a sharing equilibrium is more "likely" to arise in a competitive bottleneck environment than in a two-sided singlehoming environment. We now examine in turn the prices, the platform profits, and the surpluses of the participants.

\section{$5.1 \quad$ Prices}

We recall that in the model in which sellers multihome, platforms hold an exclusive access to their set of singlehoming buyers (the 'bottleneck'), which makes buyers valuable to extract profits on the seller side. Platforms then set monopoly prices on the multihoming side and low (and possibly even negative prices) on the singlehoming side, as has been pointed out by Armstrong (2006). In other words, we expect platforms to compete fiercely for buyers (singlehomers) and, in return, to milk sellers (multihomers). Hence, we may expect lower prices on the buyer side and higher prices on the seller side when compared to the two-sided singlehoming model. We call this the 'bottleneck effect'.

However, prices charged to sellers in the competitive bottleneck model may be low to start with and competition may well afford positive margins in the two-sided singlehoming environment. As stated in the following lemma, it depends on the parameters whether sellers pay a lower prices in the competitive bottleneck model. Comparing prices, the bottleneck effect does not necessarily dominate (there are parameter configurations that satisfy all our assumptions for either case). In addition, when moving from singlehoming to multihoming on one side, prices on both sides of the market always move in opposite directions.

Lemma 1 Allowing sellers to multihome increases the fee paid by sellers and decreases the fee paid by buyers, $m_{s}^{C B}>m_{s}^{2 S}$ and $m_{b}^{C B}<m_{b}^{2 S}$, if and only if $2 h_{s}+b_{s}+b_{b}>4 \tau_{s}-2 b_{b}$. The opposite happens - that is, $m_{s}^{C B}>m_{s}^{2 S}$ and $m_{b}^{C B}<m_{b}^{2 S}$-if and only if the left-hand and the right-hand side of the inequality are reversed-that is, $2 h_{s}+b_{s}+b_{b}<4 \tau_{s}-2 b_{b}$. Both cases are compatible with the conditions imposed on the parameters in Assumptions 1 and 2.

\footnotetext{
${ }^{8}$ It is the third line-linking the values of $h_{s}, \tau_{s}, b_{s}$, and $b_{b}$ - that will be crucial to derive our results. Given the values of these parameters, it is always possible to find values of $h_{b}$ and $\tau_{b}$ that satisfy the first two lines.
} 
Proof. The proof follows directly from computing the difference between the seller and the buyer fees in the two cases:

$$
\begin{aligned}
m_{s}^{C B}-m_{s}^{2 S} & =\frac{1}{2}\left(r_{s}+c_{s}\right)+\frac{1}{4}\left(b_{s}-b_{b}\right)-\left(c_{s}+\tau_{s}-b_{b}\right) \\
& =\frac{1}{4}\left[\left(2 h_{s}+b_{s}+b_{b}\right)-\left(4 \tau_{s}-2 b_{b}\right)\right], \\
m_{b}^{C B}-m_{b}^{2 S} & =c_{b}+\tau_{b}-\frac{b_{s}}{4 \tau_{s}}\left(3 b_{b}+b_{s}+2 r_{s}-2 c_{s}\right)-\left(c_{b}+\tau_{b}-b_{s}\right) \\
& =\frac{b_{s}}{4 \tau_{s}}\left[\left(4 \tau_{s}-2 b_{b}\right)-\left(2 h_{s}+b_{s}+b_{b}\right)\right] .
\end{aligned}
$$

As for the compatibility with Assumptions 1 and 2, we recall that conditions (FPs2S), (FPsCB) and (ShsCB) impose that $\max \left\{2 \tau_{s}, 3 \tau_{s}-b_{b}\right\}<2 h_{s}+b_{s}+b_{b}<4 \tau_{s}$. Clearly, $4 \tau_{s}-2 b_{b}<$ $4 \tau_{s}$. Moreover, if $\tau_{s}>b_{b}$, then $4 \tau_{s}-2 b_{b}>\max \left\{2 \tau_{s}, 3 \tau_{s}-b_{b}\right\}$.

Based on the intuition that sellers have to pay monopoly prices in the competitive bottleneck model and that platforms compete in this case fiercely on the multihoming side, we would consider $m_{s}^{C B}>m_{s}^{2 S}$ and $m_{b}^{C B}<m_{b}^{2 S}$ as the "natural" outcome. However, we have shown above, in the benchmark case with no cross-group effects, that the monopoly price (corresponding to the competitive bottleneck model) is always lower than the duopoly price (corresponding to the two-sided singlehoming model). This is because a drop in the fee on the seller side is more effective to expand the number of sellers when they are multihoming (in which case, the offer of a platform competes against the outside option, just as in the monopoly problem) instead of singlehoming (in which case, the offer of a platform competes against the rival's offer, just as in the standard duopoly problem). This result still holds in the borderline case in which only sellers are subject to a positive cross-group external effect $\left(b_{s}>0\right.$ and $\left.b_{b}=0\right)$ : we indeed check that $m_{s}^{C B}-m_{s}^{2 S}=\frac{1}{4}\left(2 h_{s}+b_{s}-4 \tau_{s}\right)<0$ by virtue of condition (ShsCB), which becomes $2 h_{s}<4 \tau_{s}-b_{s}$ in this particular case. It follows that the "natural" outcome can occur only if the buyers' utility increases with the number of sellers $\left(b_{b}>0\right)$.

\subsection{Platform incentives}

What are the platforms' incentives regarding single- vs multihoming? This is not a rhetorical question, as platforms may be able to use non-price strategies to prevent participants from multihoming. For instance, a platform may impose exclusivity on sellers and, thus, force them to become singlehomers. How do platform profits depend on exclusivity? To answer this question, we compare equilibrium profits:

$$
\begin{aligned}
\Pi^{C B}-\Pi^{2 S} & =\left[\left(m_{b}^{C B}-c_{b}\right) n_{b}^{C B}+\left(m_{s}^{C B}-c_{s}\right) n_{s}^{C B}\right]-\left[\left(m_{b}^{2 S}-c_{b}\right) n_{b}^{2 S}+\left(m_{s}^{2 S}-c_{s}\right) n_{s}^{2 S}\right] \\
& =\frac{1}{2}\left(m_{s}^{C B}-m_{s}^{2 S}\right)\left(1-\frac{b_{s}}{\tau_{s}}\right)+\left(n_{s}^{C B}-\frac{1}{2}\right)\left(m_{s}^{C B}-c_{s}\right) .
\end{aligned}
$$

where the second line uses our previous results, namely $n_{b}^{C B}=n_{b}^{2 S}=n_{s}^{2 S}=\frac{1}{2}, n_{s}^{C B}>1 / 2$ and $m_{b}^{C B}-m_{b}^{2 S}=-\left(b_{s} / \tau_{s}\right)\left(m_{s}^{C B}-m_{s}^{2 S}\right)$. We see that if, for instance, sellers pay a higher fee in the competitive bottleneck case and this fee is larger than marginal cost $\left(m_{s}^{C B}>m_{s}^{2 S}\right.$ and $m_{s}^{C B}>c_{s}$ ) while $\tau_{s}>b_{s}$, then all terms are positive, meaning that platforms make higher 
profits when sellers can multihome. Conversely, still in the case where $\tau_{s}>b_{s}$, if platforms subsidize sellers in the competitive bottleneck case $\left(m_{s}^{C B}<c_{s}\right)$ and set a lower fee than in the two-sided singlehoming case $\left(m_{s}^{C B}<m_{s}^{2 S}\right)$, then all terms are negative and platforms prefer to prevent sellers from multihoming.

To formalize this intuition, we use the values of $m_{s}^{C B}, m_{s}^{2 S}$ and $n_{s}^{C B}$ to compute

$$
\Pi^{C B}-\Pi^{2 S}=\frac{4 h_{s}^{2}+8\left(b_{b}+b_{s}\right) \tau_{s}-8 \tau_{s}^{2}-\left(b_{b}^{2}+6 b_{b} b_{s}+b_{s}^{2}\right)}{16 \tau_{s}} .
$$

A sufficient condition for $\Pi^{C B}>\Pi^{2 S}$ is $8\left(b_{b}+b_{s}\right) \tau_{s}-8 \tau_{s}^{2}-\left(b_{b}^{2}+6 b_{b} b_{s}+b_{s}^{2}\right)>0$ (as we assume $\left.h_{s}>0\right)$. This polynomial in $\tau_{s}$ has two positive roots, $\left(b_{b}+b_{s}\right) / 2 \pm \sqrt{2}\left(b_{b}-b_{s}\right) / 4$, and is positive if $\tau_{s}$ is comprised between the two roots. Otherwise, we have that

$$
\Pi^{C B}<\Pi^{2 S} \Leftrightarrow h_{s}<\frac{1}{2} \sqrt{8 \tau_{s}^{2}-8\left(b_{b}+b_{s}\right) \tau_{s}+\left(b_{b}^{2}+6 b_{b} b_{s}+b_{s}^{2}\right)} \equiv h_{s}^{\Pi} .
$$

Recalling that $2 h_{s}<4 \tau_{s}-b_{b}-b_{s}$ according to condition (ShsCB), we observe that the latter inequality is always satisfied if $\tau_{s}<\sqrt{b_{b} b_{s} / 2} .{ }^{9}$ We record our results in the following lemma.

Lemma 2 If $-\sqrt{2}\left|b_{b}-b_{s}\right|<4 \tau_{s}-2 b_{b}-2 b_{s}<\sqrt{2}\left|b_{b}-b_{s}\right|$, then platforms are always willing to allow sellers to multihome. In contrast, if $\tau_{s}<\sqrt{b_{b} b_{s} / 2}$, then platforms are always willing to prevent sellers from multihoming. Outside this regions of parameters, platforms prefer to allow sellers to multihome if and only if $h_{s}>h_{s}^{\Pi}$.

\subsection{Participants' surpluses}

In this subsection we compare the aggregate surplus of buyers and sellers in the two environments. In particular, we want to know to whether buyers' and sellers' preferences aligned or misaligned regarding the multihoming of sellers. Our previous discussion about equilibrium fees points at a major source of misalignment, as fees move in opposite directions: when sellers pay lower fees in the competitive bottleneck case, buyers pay lower fees in the two-sided singlehoming case, and vice versa. However, participants also care about the number of agents of the other group they can interact with, and these numbers also differ in the two environments. First, as $n_{s}^{C B}>1 / 2$, there are more sellers active on a platform under multihoming than under singlehoming, thus adding value to participation on the buyer side. Second, multihoming sellers have access to all buyers, which may positively affect their surplus (even if they pay twice the fees and the transportation costs). Thus, we need to examine how the effects of price and participation balance one another.

Buyers. For buyers, we have $v_{b}^{C B}-v_{b}^{2 S}=\left(n_{s}^{C B}-1 / 2\right) b_{b}-\left(m_{b}^{C B}-m_{b}^{2 S}\right)$. The first term is the participation effect and is clearly positive, as multihoming brings more sellers on each

\footnotetext{
${ }^{9}$ It is readily checked that $\tau_{s}<\sqrt{b_{b} b_{s} / 2}<\min \left\{\left(b_{b}+b_{s}\right) / 2-\sqrt{2}\left(b_{b}-b_{s}\right) / 4,\left(b_{b}+b_{s}\right) / 2+\sqrt{2}\left(b_{b}-b_{s}\right) / 4\right\}$. Recall also that parameters must satisfy condition (Soc2S), i.e., $4 \tau_{b} \tau_{s}>\left(b_{b}+b_{s}\right)^{2}$, which can be rewritten as $2 \tau_{s}^{2}>\left(\left(b_{b}+b_{s}\right)^{2} /\left(2 \tau_{b}\right)\right)^{2}$. This condition is compatible with $\tau_{s}<\sqrt{b_{b} b_{s} / 2}$ as long as $\tau_{b}>\left(b_{b}+b_{s}\right)^{2} /\left(2 \sqrt{b_{b} b_{s}}\right)$.
} 
platform; the second is the price effect and, as we have seen above, it can be either negative (if $m_{b}^{C B}>m_{b}^{2 S}$ ) or positive. At the aggregate level, $C S^{C B}=v_{b}^{C B}-\tau_{b} / 4$ and $C S^{2 S}=v_{b}^{2 S}-\tau_{b} / 4$ imply that $C S^{C B}-C S^{2 S}=v_{b}^{C B}-v_{b}^{2 S}$. Using the definition of $n_{s}^{C B}$ and the expression derived above for $m_{b}^{C B}-m_{b}^{2 S}$, we can write

$$
C S^{C B}-C S^{2 S}=\left[\frac{1}{4 \tau_{s}}\left(2 h_{s}+b_{b}+b_{s}\right)-\frac{1}{2}\right] b_{b}-\frac{b_{s}}{4 \tau_{s}}\left[\left(4 \tau_{s}-2 b_{b}\right)-\left(2 h_{s}+b_{s}+b_{b}\right)\right] .
$$

It is clear that the participation effect (the first term) increases with the strength of the crossgroup effect that sellers exert on buyers $\left(b_{b}\right)$, and with the equilibrium number of sellers in the competitive bottleneck case, which is itself an increasing function of $h_{s}$ and a decreasing function of $\tau_{s}$ (more sellers decide to multihome when their intrinsic benefits are larger and when transportation costs are smaller). It can be checked that an increase in $b_{b}$ or $h_{s}$, or a decrease in $\tau_{s}$ also reduce the importance of the price effect (the second term), thereby making it unambiguously more likely that $C S^{C B}>C S^{2 S} .{ }^{10}$

Developing the previous expression, we find

$$
C S^{C B}>C S^{2 S} \Leftrightarrow h_{s}>\frac{4 \tau_{s}-b_{s}-b_{b}}{2}-b_{b} \frac{b_{s}+\tau_{s}}{b_{s}+b_{b}} \equiv h_{s}^{b} .
$$

It is readily checked that the latter inequality is more likely to be satisfied when $b_{b}$ increases or when $\tau_{s}$ decreases, which confirm our previous intuition. Recall that conditions (FPs2S), (FPsCB) and (ShsCB), together with $h_{s}>0$, impose

$$
h_{s}^{\min } \equiv \max \left\{\frac{1}{2}\left(2 \tau_{s}-b_{s}-b_{b}\right), \frac{1}{2}\left(3 \tau_{s}-b_{s}-2 b_{b}\right), 0\right\}<h_{s}<h_{s}^{\max } \equiv \frac{1}{2}\left(4 \tau_{s}-b_{s}-b_{b}\right) .
$$

It is clear that $h_{s}^{b}$ is less than the upper bound. Hence, there always exist admissible values of $h_{s}$ that are sufficiently large for consumers to prefer that sellers be allowed to multihome. However, parameters may be such that $h_{s}^{b}$ is also less than the lower bound, implying that buyers prefer sellers to multihome for any admissible value of $h_{s}$. This is so, for instance, if $b_{b}>b_{s}$, i.e., if buyers value more the interaction with sellers than vice versa. We formalize these findings in the next lemma (which is proved in Appendix 8.1).

Lemma 3 If $b_{b}>b_{s}$ or if $b_{s}>b_{b}$ and $\tau_{s}<\left(b_{b}^{2}+4 b_{b} b_{s}+b_{s}^{2}\right) /\left(2\left(b_{b}+2 b_{s}\right)\right) \equiv \tau_{s}^{\min }$, then buyers are always better off when sellers are allowed to multihome $\left(C S^{C B}>C S^{2 S}\right)$. Otherwise (i.e., for $b_{s}>b_{b}$ and $\left.\tau_{s}>\tau_{s}^{\min }\right)$, they may prefer that sellers be forced to singlehome $\left(C S^{2 S}>C S^{C B}\right)$ for small values of $h_{s}=r_{s}-c_{s}$ (i.e., for $h_{s}^{\min }<h_{s}<h_{s}^{b}$ ).

In summary, Lemma 3 shows that we can expect consumers to welcome the possibility for sellers to multihome, because it gives them access to a larger set of sellers with whom they can interact, and it may even lead platforms to charge them lower fees. This is all the more likely that they value a lot the presence of sellers and that sellers find it profitable to multihome. We provide sufficient conditions for this to be only possible outcome. However, it may happen that platforms charge higher fees to buyers when sellers multihome than when they singlehome, and that this negative price effect outweighs the positive participation effect - if this is the case, buyers prefer that platforms actually prevent seller multihoming.

\footnotetext{
${ }^{10}$ The net effect of a change in $b_{s}$ is ambiguous.
} 
Sellers. For singlehoming sellers, we have $v_{s}^{C B}-v_{s}^{2 S}=m_{s}^{2 S}-m_{s}^{C B}$; here, there is no participation effect, as buyers equally split between the two platforms in both environments; and we have seen above that the price difference can go both ways. As for multihoming sellers, we focus on the one located at the middle of the Hotelling line for whom the surplus difference is equal to $2 v_{s}^{C B}-\tau_{s}-\left(v_{s}^{2 S}-\tau_{s} / 2\right)=r_{s}+\left(b_{s}-\tau_{s}\right) / 2-\left(2 m_{s}^{C B}-m_{s}^{2 S}\right)$. Developing the latter expression, we find that this seller is better off in the competitive bottleneck environment than in the singlehoming environment if and only if $\tau_{s}>b_{b}{ }^{11}$

At the aggregate level, we recall the expressions derived in Sections 3 and 4:

$$
\begin{aligned}
P S^{C B} & =\frac{1}{\tau_{s}}\left(v_{s}^{C B}\right)^{2}=\frac{1}{16 \tau_{s}}\left(b_{b}+b_{s}+2 h_{s}\right)^{2}, \\
P S^{2 S} & =v_{s}^{2 S}-\frac{1}{4} \tau_{s}=h_{s}-\frac{5}{4} \tau_{s}+\frac{1}{2} b_{s}+b_{b} .
\end{aligned}
$$

It follows that

$$
P S^{C B}>P S^{2 S} \Leftrightarrow 4 h_{s}^{2}-4\left(4 \tau_{s}-b_{b}-b_{s}\right) h_{s}+20 \tau_{s}^{2}-8\left(2 b_{b}+b_{s}\right) \tau_{s}+\left(b_{b}+b_{s}\right)^{2}>0 .
$$

If $\tau_{s}>2 b_{b}$, this polynomial in $h_{s}$ has no real root in which case it can be shown that it is positive everywhere, meaning that $P S^{C B}>P S^{2 S}$. Otherwise, for $\tau_{s}<2 b_{b}$, the polynomial has two real roots. The larger roots is equal to $\frac{1}{2}\left(4 \tau_{s}-b_{s}-b_{b}\right)+\sqrt{\tau_{s}\left(2 b_{b}-\tau_{s}\right)}$. Recalling that our parameter restrictions impose that $h_{s}<h_{s}^{\max }=\frac{1}{2}\left(4 \tau_{s}-b_{s}-b_{b}\right)$, we immediately see that this root lies above the upper bound of the admissible range. As for the lower root, we denote it

$$
h_{s}^{s} \equiv \frac{1}{2}\left(4 \tau_{s}-b_{s}-b_{b}\right)-\sqrt{\tau_{s}\left(2 b_{b}-\tau_{s}\right)} .
$$

As established in the following lemma (see Appendix 8.2 for the formal proof), the inequality $h_{s}^{s}>h_{s}^{\mathrm{min}}$ must hold unless $\tau_{s}$ is sufficiently small. Then, we have that $P S^{C B}>P S^{2 S}$ for $h_{s}<h_{s}^{s}$ and $P S^{C B}<P S^{2 S}$ otherwise. In the cases in which $h_{s}^{s}<h_{s}^{\min }$, we have that $P S^{C B}<P S^{2 S}$ for all admissible parameter configurations.

Lemma 4 If $\tau_{s}>2 b_{b}$, then sellers are always better off when they are allowed to multihome $\left(P S^{C B}>P S^{2 S}\right)$. In contrast, for sufficiently small values of $\tau_{s}$, sellers are always better off when they are prevented from multihoming $\left(P S^{C B}<P S^{2 S}\right)$. For intermediate values of $\tau_{s}$, sellers prefer to multihome if $h_{s}<h_{s}^{s}$, and to singlehome otherwise.

According to Lemma 4, sellers' preferences regarding multihoming crucially depend on the ratio between the degree of platform differentiation, $\tau_{s}$, and the strength of cross-group effects that sellers exert on buyers, $b_{b}$ : the larger this ratio - that is, the larger $\tau_{s}$ and/or the smaller $b_{b}$ - the more likely it is that sellers are better off in the competitive bottleneck case. This stands in sharp contrast with what we observed for buyers, who are more likely to prefer that sellers singlehome when $\tau_{s}$ increase and/or $b_{b}$ decrease. Thus, we have identified here an important

\footnotetext{
${ }^{11}$ Recall that under this condition, full participation of sellers is guaranteed in the competitive bottleneck case if full participation is guaranteed in the two-sided singlehoming case-i.e., condition (FPs2S) implies condition $(\mathrm{FBsCP})$.
} 
source of divergence between the preferences of buyers and sellers. However, this does not exclude the existence of parameter configurations such that buyers and sellers agree, as we discuss in the following subsection.

\subsection{Surplus comparisons: The complete picture}

To close this section, we superimpose the results of Lemmas $2-4$, so as to measure the extent to which platforms, buyers and sellers agree or disagree with respect to the benefits from seller multihoming. There are a priori eight possible scenarios. In the next proposition, we eliminate three of them by proving a clear divergence between buyers on one side, and sellers and platforms on the other side (the proof is relegated to Appendix 8.3).

Proposition 1 Whenever buyers prefer that sellers be forced to singlehome $\left(C S^{2 S}>C S^{C B}\right)$, both sellers and platforms prefer the opposite $\left(P S^{C B}>P S^{2 S}\right.$ and $\left.\Pi^{C B}>\Pi^{2 S}\right)$.

Proposition 1 leaves us with four other scenarios. The first two scenarios correspond to the conventional wisdom: $C S^{C B}>C S^{2 S}$ and $P S^{2 S}>P S^{C B}$; that is, the possibility of multihoming is beneficial for the side that continues to singlehome, but harmful for the side that is allowed to multihome. Then, platforms may have higher profits in either environment: they will please sellers if they choose to impose exclusivity, and please buyers otherwise.

In the last two scenarios, both buyers and sellers are better off in the competitive bottleneck environment: $C S^{C B}>C S^{2 S}$ and $P S^{C B}>P S^{2 S}$. Again, platforms may prefer one or the other environment. Here, if they impose exclusivity (which can only occur if $b_{b}>b_{s}$ ), they hurt both groups; otherwise, the possibility of multihoming for sellers is welfare improving as it makes all parties better off. We summarize our findings in the next proposition.

Proposition 2 (1) Whenever platforms find it preferable to impose exclusivity, they necessarily hurt at least one group of participants. (2) It is possible that buyers, sellers and platforms are all better off when sellers are allowed to multihome.

Proof. The first part directly follows from Proposition 1, as buyers and sellers never agree that exclusivity would make them all better off. To show the existence of the other configurations, we build numerical examples. First, take $b_{b}>b_{s}$ and $\tau_{s}>2 b_{b}$, so that respectively buyers and sellers always prefer the competitive bottleneck environment. Set $b_{b}=40, b_{s}=10$ and $\tau_{s}=85$. Then, we have $h_{s}^{\Pi}=83.516$ while $h_{s}^{\min }=82.5$ and $h_{s}^{\max }=145$. For $h_{s}=83$, we check that $\Pi^{C B}-\Pi^{2 S}=-43 / 170<0$, in which case platforms would impose exclusivity, thereby hurting both buyers and sellers. In contrast, for $h_{s}=85$, we have $\Pi^{C B}-\Pi^{2 S}=25 / 34>0$, in which case all parties agree that the competitive bottleneck environment is preferable.

We summarize our results in Table 1 and Figure 1. Table 1 shows the five possible combinations of preferences for the three parties - buyers, sellers, and platforms ('CP' indicates a preference for the competitive bottleneck case, ' $2 \mathrm{~S}$ ' for the two-sided singlehoming case). In the 
last column of the table, we indicate the zones of Figure 1 that correspond to the various combinations (the left panel corresponds to the case in which $b_{b}>b_{s}$ and the right panel, to the case in which $\left.b_{s}>b_{b}\right)$.

\begin{tabular}{cccc}
\hline \hline Buyers & Sellers & Platforms & Zones in Figure 1 \\
\hline $\mathrm{CB}$ & $2 \mathrm{~S}$ & $2 \mathrm{~S}$ & 1,5 \\
$\mathrm{CB}$ & $2 \mathrm{~S}$ & $\mathrm{CB}$ & 2,6 \\
$\mathrm{CB}$ & $\mathrm{CB}$ & $\mathrm{CB}$ & 3,7 \\
$\mathrm{CB}$ & $\mathrm{CB}$ & $2 \mathrm{~S}$ & 4 \\
$2 \mathrm{~S}$ & $\mathrm{CB}$ & $\mathrm{CB}$ & 8 \\
\hline \hline
\end{tabular}

Table 1. Preferred market environments
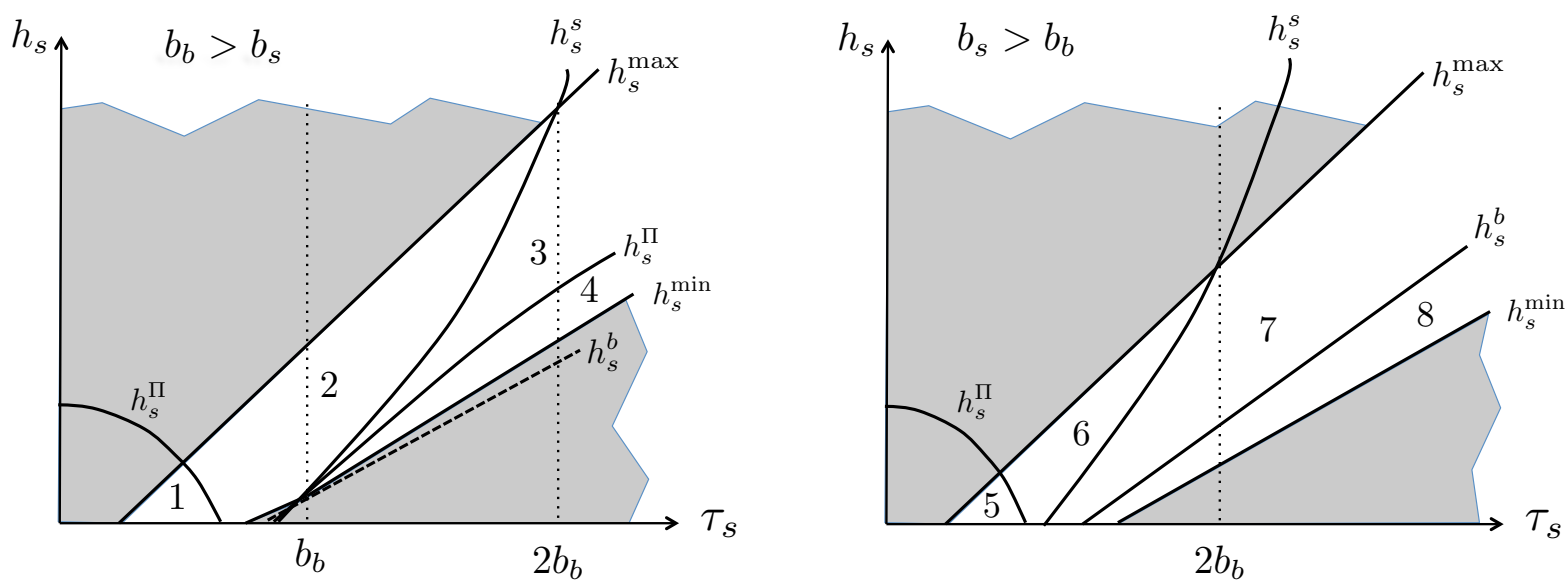

Figure 1: Surplus effects of seller multihoming

We conclude that without further information, a competition authority or regulator cannot know whether allowing multihoming on one side (with the other side singlehoming) leads to higher or lower net surpluses on either side. It is, therefore, a priori not possible to say whether the side that changes its behavior from singlehoming to multihoming (or reverse) benefits or suffers from this change of behavior.

\section{Extension: Stand-alone benefits when multihoming}

We propose here a more general formulation of the competitive bottleneck problem by assuming that a multihoming seller enjoys a total stand-alone benefit equal to $(1+\rho) r_{s}$. We let the parameter $\rho$ take any value between 0 and 1 to cover any situation regarding the services that give rise to the stand-alone utility. At one extreme $(\rho=1)$, we have the case considered so far in this paper: platforms provide completely differentiated services. At the other extreme $(\rho=0)$, we have the case analyzed by Armstrong and Wright (2007): platforms provide exactly the same services, so that joining a second platform does not generate any extra stand-alone 
benefit. In this section, we consider the whole spectrum between these two extremes. Our goal is to show that our results still hold under this more general formulation and that our main message is even reinforced - that is, the conventional wisdom about the effects of multihoming cannot be trusted. As the analysis follows the same steps as in Section 4, we skip most of the developments.

Derivation of equilibrium. The sellers who are indifferent between visiting platform $i(i \in$ $\{1,2\})$ and visiting both platforms are now identified by

$$
x_{1 m}=1-\frac{1}{\tau_{s}}\left(\rho r_{s}+n_{b}^{2} b_{s}-m_{s}^{2}\right) \text { and } x_{2 m}=\frac{1}{\tau_{s}}\left(\rho r_{s}+n_{b}^{1} b_{s}-m_{s}^{1}\right) .
$$

As above, we assume that $0<x_{1 m}<x_{2 m}<1$, so that $n_{s}^{1}=x_{2 m}$ and $n_{s}^{2}=1-x_{1 m}$. Noting that nothing changes for buyers, we can proceed in the same way as in Section 4: we solve for buyers' and sellers' participation levels and then use these expressions to derive the platforms' profits as functions of the four fees. Solving the systems of the four first-order conditions, we find the following equilibrium fees and equilibrium participation, where $h_{s, \rho}$ stands for $\rho r_{s}-c_{s}$ (note that $h_{s, \rho}=h_{s}=r_{s}-c_{s}$ for $\rho=1$ ):

$$
\begin{aligned}
m_{b}^{c b} & =c_{b}+\tau_{b}-\frac{b_{s}}{4 \tau_{s}}\left(b_{s}+3 b_{b}+2 h_{s, \rho}\right), \\
m_{s}^{c b} & =c_{s}+\frac{1}{2} h_{s, \rho}+\frac{1}{4}\left(b_{s}-b_{b}\right), \\
n_{b}^{c b} & =\frac{1}{2} \text { and } n_{s}^{c b}=\frac{1}{4 \tau_{s}}\left(b_{b}+b_{s}+2 h_{s, \rho}\right) .
\end{aligned}
$$

From there, we can compute the equilibrium net surplus of sellers and buyers (gross of transportation costs) as:

$$
\begin{aligned}
v_{s}^{s h} & =\frac{1}{4}\left(b_{b}+b_{s}\right)+\frac{1}{2}\left((2-\rho) r_{s}-c_{s}\right), \\
v_{s}^{m h} & =\frac{1}{2}\left(b_{b}+b_{s}\right)+\left(r_{s}-c_{s}\right), \\
v_{b}^{c b} & =\frac{1}{4 \tau_{s}}\left(b_{b}^{2}+4 b_{s} b_{b}+b_{s}^{2}+2\left(b_{b}+b_{s}\right) h_{s, \rho}\right)+h_{b}-\tau_{b},
\end{aligned}
$$

where $v_{s}^{s h}$ denotes the surplus for singlehoming sellers (i.e., sellers located between 0 and $1-n_{s}^{c b}$, who choose to visit platform 1 only, and sellers located between $n_{s}^{c b}$ and 1, who choose to visit platform 2 only) and $v_{s}^{m h}$ the surplus for multihoming sellers (i.e., sellers located between $1-n_{s}^{c b}$ and $\left.n_{s}^{c b}\right)$. Note that $v_{s}^{m h}=2 v_{s}^{s h}-(1-\rho) r_{s}$ - that is multihoming sellers earn less than twice the surplus of singlehoming sellers because of the possible duplication between the stand-alone benefits provided by the two platforms. For the special case $\rho=1$ (no duplication), $v_{s}^{m h}=2 v_{s}^{s h}$, as postulated in Section 4 .

Aggregated over all buyers, in equilibrium, buyers' surplus is

$$
C S^{c b}=\frac{1}{4 \tau_{s}}\left(\left(b_{b}+b_{s}\right)\left(b_{b}+b_{s}+2 h_{s, \rho}\right)+2 b_{b} b_{s}\right)+h_{b}-\frac{5}{4} \tau_{b} .
$$

Aggregated over all sellers, in equilibrium, sellers' surplus is

$$
\begin{aligned}
P S^{c b} & =\int_{0}^{x_{1 m}^{c b}}\left(v_{s}^{s h}-\tau_{s} x\right) d x+\int_{x_{1 m}^{c b}}^{x_{2 m}^{c b}}\left(v_{s}^{m h}-\tau_{s}\right) d x+\int_{x_{2 m}^{c b}}^{1}\left(v_{s}^{s h}-\tau_{s}(1-x)\right) d x \\
& =\frac{1}{16 \tau_{s}}\left(b_{b}+b_{s}+2 h_{s, \rho}\right)^{2}+(1-\rho) r_{s} .
\end{aligned}
$$


Finally, the platforms' equilibrium profits are computed as

$$
\Pi^{c b}=\frac{1}{16 \tau_{s}}\left(8 \tau_{b} \tau_{s}-\left(b_{s}^{2}+b_{b}^{2}+6 b_{s} b_{b}\right)+4 h_{s, \rho}^{2}\right) .
$$

It is easily seen that the conditions of Assumption 2 guarantee that this equilibrium holds for any value of $\rho$ (the conditions become more stringent as $\rho$ increases).

Multihoming vs. singlehoming. We observe that the expressions for the equilibrium prices, participations, buyers' surplus and platforms' profits are isomorphic to the ones obtained in Section 4: we just need to replace $h_{s}$ by $h_{s, \rho}$. As $h_{s, \rho} \leq h_{s}$, we can immediately conclude that

$$
m_{b}^{c b} \geq m_{b}^{C B}, m_{s}^{c b} \leq m_{s}^{C B}, n_{s}^{c b} \leq n_{s}^{C B}, C S^{c b} \leq C S^{C B} \text {, and } \Pi^{c b} \leq \Pi^{C B} \text {. }
$$

Thus, the more similar the services provided by the platforms (i.e., the smaller $\rho$ ), the higher the fee charged to buyers and the lower the fee charged to sellers; there are also fewer multihoming sellers, which results in a lower surplus for buyers and lower profits for platforms. The impact on sellers is less obvious, since an additional term appears in $P S^{c b}$. We compute

$$
\begin{aligned}
P S^{c b}-P S^{C B} & =\frac{1}{4 \tau_{s}}\left(b_{b}+b_{s}+h_{s, \rho}+h_{s}\right)\left(h_{s, \rho}-h_{s}\right)+(1-\rho) r_{s} \\
& =(1-\rho) r_{s}\left(1-\frac{1}{4 \tau_{s}}\left(b_{b}+b_{s}+h_{s, \rho}+h_{s}\right)\right) \\
& =(1-\rho) r_{s}\left(1-n_{s}^{C B}+(1-\rho) \frac{r_{s}}{4 \tau_{s}}\right) \geq 0 .
\end{aligned}
$$

Hence, sellers obtain a higher surplus as $\rho$ decreases.

The previous results suggest than when comparing the competitive bottleneck and the twosided singlehoming environments, it is more likely, as $\rho$ decreases, that sellers prefer the former, while buyers and platforms prefer the latter. Hence, a larger duplication of stand-alone benefits (i.e., a lower $\rho$ ) further undermines the conventional wisdom, according to which the possibility of multihoming should hurt the side that can multihome (here, sellers) while benefiting the other side.

\section{Conclusion}

In this paper, we have reconsidered the classic Armstrong (2006) two-sided platform setting with the aim to analyze the impact of multihoming on one side on prices, platform profits, and buyer and seller surplus. The competitive bottleneck world is described as a world in which the multihoming side has to pay monopoly prices and platforms compete on the singlehoming side. However, this does not imply that the multihoming side were to pay lower prices if it could not multihome. We recall the three main insights regarding the surplus platforms, buyers, and sellers obtain. (i) Whenever buyers prefer that sellers be forced to singlehome, both sellers and platforms prefer the opposite. (ii) Whenever platforms find it preferable to impose exclusivity, they necessarily hurt at least one group of participants. (iii) It is possible that buyers, sellers 
and platforms are all better off when sellers are allowed to multihome. All our findings are easily reformulated if the buyer instead of the seller side is the side that may be able to multihome.

Future work may look into alternative settings to address the effect of multihoming. This appears to be a worthwhile endeavour given the inclination of some competition authorities and regulators to encourage and facilitate multihoming.

\section{Appendix}

\subsection{Proof of Lemma 3}

We first note that

$$
\begin{aligned}
h_{s}^{b} & >\frac{1}{2}\left(2 \tau_{s}-b_{s}-b_{b}\right) \Leftrightarrow \tau_{s}>b_{b}, \\
h_{s}^{b} & >\frac{1}{2}\left(3 \tau_{s}-b_{s}-2 b_{b}\right) \Leftrightarrow\left(\tau_{s}-b_{b}\right)\left(b_{s}-b_{b}\right)>0, \\
h_{s}^{b} & >0 \Leftrightarrow \tau_{s}>\frac{b_{b}^{2}+4 b_{b} b_{s}+b_{s}^{2}}{2\left(b_{b}+2 b_{s}\right)} \equiv \tau_{s}^{\min } .
\end{aligned}
$$

(1) Take $b_{b}>b_{s}$. (1a) If $\tau_{s}>b_{b}$, then $h_{s}^{\min }=\frac{1}{2}\left(3 \tau_{s}-b_{s}-2 b_{b}\right)$ and we see from the middle line above that $h_{s}^{b}<h_{s}^{\min }$. (1b) If $\left(b_{b}+b_{s}\right) / 2<\tau_{s}<b_{b}$, then $h_{s}^{\min }=\frac{1}{2}\left(2 \tau_{s}-b_{s}-b_{b}\right)$ and we see from the top line above that $h_{s}^{b}<h_{s}^{\min }$. (1c) If $\tau_{s}<\left(b_{b}+b_{s}\right) / 2$, then $h_{s}^{\min }=0$ and as $\tau_{s}^{\min }>\left(b_{b}+b_{s}\right) / 2$ when $b_{b}>b_{s}$, we have again, from the bottom line above, that $h_{s}^{b}<h_{s}^{\min }$. (2) Take $b_{s}>b_{b}$. (2a) If $\tau_{s}>\left(b_{s}+2 b_{b}\right) / 3$, then $h_{s}^{\min }=\frac{1}{2}\left(3 \tau_{s}-b_{s}-2 b_{b}\right)$ and as $\left(b_{s}+2 b_{b}\right) / 3>b_{b}$ when $b_{s}>b_{b}$, we have that $h_{s}^{b}<h_{s}^{\text {min }}$ from the middle line above. (2b) If $\tau_{s}<\left(b_{s}+2 b_{b}\right) / 3$, $h_{s}^{\min }=0$ and as $\tau_{s}^{\min }<\left(b_{s}+2 b_{b}\right) / 3$, we have that $h_{s}^{b}>h_{s}^{\min }$ for $\tau_{s}<\tau_{s}^{\min }$.

\subsection{Proof of Lemma 4}

We first establish the sign of $C \equiv 20 \tau_{s}^{2}-8\left(2 b_{b}+b_{s}\right) \tau_{s}+\left(b_{b}+b_{s}\right)^{2}$. This quadratic form in $\tau_{s}$ has real roots provided that $11 b_{b}^{2}+6 b_{b} b_{s}-b_{s}^{2}>0$, which is guaranteed if $b_{s}<7.45 b_{b}$. If $b_{s}>7.45 b_{b}$, $C>0$ for all $\tau_{s}$. If $b_{s}<7.45 b_{b}$, then the two roots, which we note $\tau_{s}^{-}$and $\tau_{s}^{+}$, are such that

$0<\tau_{s}^{-}=\frac{1}{5}\left(2 b_{b}+b_{s}\right)-\frac{1}{10} \sqrt{11 b_{b}^{2}+6 b_{b} b_{s}-b_{s}^{2}}<\tau_{s}^{+}=\frac{1}{5}\left(2 b_{b}+b_{s}\right)+\frac{1}{10} \sqrt{11 b_{b}^{2}+6 b_{b} b_{s}-b_{s}^{2}}<2 b_{b}$.

So, $C$ is positive for $\tau_{s}<\tau_{s}^{-}$or $\tau_{s}>\tau_{s}^{+}$, and negative otherwise. We now rewrite the condition $P S^{C B}>P S^{2 S}$ as $4 h_{s}^{2}-4\left(4 \tau_{s}-b_{b}-b_{s}\right) h_{s}+C>0$. For this quadratic form to have real roots, we need $4\left(4 \tau_{s}-b_{b}-b_{s}\right)^{2}-4 C=16 \tau_{s}\left(2 b_{b}-\tau_{s}\right)>0$, or $\tau_{s}<2 b_{b}$. If $\tau_{s}>2 b_{b}$, then $C>0$, implying that $P S^{C B}>P S^{2 S}$. Consider now the case where $\tau_{s}<2 b_{b}$. There are then two positive roots: $h_{s}^{s} \equiv \frac{1}{2}\left(4 \tau_{s}-b_{s}-b_{b}\right)-\sqrt{\tau_{s}\left(2 b_{b}-\tau_{s}\right)}$ and $\frac{1}{2}\left(4 \tau_{s}-b_{s}-b_{b}\right)+\sqrt{\tau_{s}\left(2 b_{b}-\tau_{s}\right)}>h_{s}^{\max }$. Clearly, $h_{s}^{s}>0$ if and only if $C>0$. So, in case $h_{s}^{s}>h_{s}^{\min }$, then $P S^{C B}>P S^{2 S}$ if $h_{s}<h_{s}^{s}$, whereas $P S^{C B}<P S^{2 S}$ otherwise. If $h_{s}^{s}<0$, then $P S^{C B}<P S^{2 S}$ for all admissible $h_{s}>h_{s}^{s}$. We still need to compare $h_{s}^{s}$ to $h_{s}^{\min }$. We have that

$$
\begin{aligned}
& h_{s}^{s}>\frac{1}{2}\left(2 \tau_{s}-b_{s}-b_{b}\right) \Leftrightarrow \tau_{s}>b_{b}, \\
& h_{s}^{s}>\frac{1}{2}\left(3 \tau_{s}-b_{s}-2 b_{b}\right) \Leftrightarrow\left(\tau_{s}-b_{b}\right)\left(5 \tau_{s}-b_{b}\right)>0 .
\end{aligned}
$$


(1) Take $b_{b}>b_{s}$. (1a) If $\tau_{s}>b_{b}$, then $h_{s}^{\min }=\frac{1}{2}\left(3 \tau_{s}-b_{s}-2 b_{b}\right)$ and we see from the bottom line above that $h_{s}^{s}>h_{s}^{\min }$. (1b) If $\left(b_{b}+b_{s}\right) / 2<\tau_{s}<b_{b}$, then $h_{s}^{\min }=\frac{1}{2}\left(2 \tau_{s}-b_{s}-b_{b}\right)$ and we see from the top line above that $h_{s}^{s}<h_{s}^{\min }$. (1c) If $\tau_{s}<\left(b_{b}+b_{s}\right) / 2$, then $h_{s}^{\min }=0$; as $\tau_{s}^{-}<\left(b_{b}+b_{s}\right) / 2<\tau_{s}^{+}$, we have that $h_{s}^{s}>h_{s}^{\min }$ for $\tau_{s}<\tau_{s}^{-}$and $h_{s}^{s}<h_{s}^{\min }$ for $\tau_{s}^{-}<\tau_{s}<$ $\left(b_{b}+b_{s}\right) / 2$. (2) Take $b_{s}>b_{b}$. (2a) If $\tau_{s}>\left(b_{s}+2 b_{b}\right) / 3$, then $h_{s}^{\min }=\frac{1}{2}\left(3 \tau_{s}-b_{s}-2 b_{b}\right)$ and as $\left(b_{s}+2 b_{b}\right) / 3>b_{b}$ when $b_{s}>b_{b}$, we have that $h_{s}^{s}>h_{s}^{\text {min }}$ from the bottom line above. (2b) If $\tau_{s}<\left(b_{s}+2 b_{b}\right) / 3, h_{s}^{\min }=0$; as $\tau_{s}^{-}<\tau_{s}^{+}<\left(b_{s}+2 b_{b}\right) / 3$, we have that $h_{s}^{s}>h_{s}^{\min }$ for $\tau_{s}<\tau_{s}^{-}$ and $\tau_{s}^{+}<\tau_{s}<\left(b_{s}+2 b_{b}\right) / 3$, whereas $h_{s}^{s}<h_{s}^{\min }$ for $\tau_{s}^{-}<\tau_{s}<\tau_{s}^{+}$. Collecting the previous results, we have that $h_{s}^{s}<h_{s}^{\text {min }}$ in which case $P S^{C B}<P S^{2 S}$ for all admissible parameters if either $b_{b}>b_{s}$ and $\tau_{s}^{-}<\tau_{s}<b_{b}$ or $b_{s}>b_{b}$ and $\tau_{s}^{-}<\tau_{s}<\tau_{s}^{+}$.

\subsection{Proof of Proposition 1}

From Lemma 3, we know that for buyers to prefer that sellers singlehome, we must have $b_{s}>b_{b}$, $\tau_{s}>\tau_{s}^{\min }$, and $h_{s}^{\min }<h_{s}<h_{s}^{b}$. (1) We first show that in that case, sellers prefer the possibility of multihoming. Suppose not. We know from Lemma 4 that for sellers to prefer two-sided singlehoming, we must have $h_{s}>h_{s}^{s}$. So, it must be that $h_{s}^{s}<h_{s}<h_{s}^{b}$. However, $h_{s}^{b}>h_{s}^{s}$ is incompatible with $b_{s}>b_{b}$ and $\tau_{s}>\tau_{s}^{\min }$ :

$$
\begin{aligned}
h_{s}^{b} & >h_{s}^{s} \Leftrightarrow \frac{1}{2}\left(4 \tau_{s}-b_{s}-b_{b}\right)-b_{b} \frac{b_{s}+\tau_{s}}{b_{s}+b_{b}}>\frac{1}{2}\left(4 \tau_{s}-b_{s}-b_{b}\right)-\sqrt{\tau_{s}\left(2 b_{b}-\tau_{s}\right)} \\
& \Leftrightarrow \tau_{s}\left(2 b_{b}-\tau_{s}\right)-\left(b_{b} \frac{b_{s}+\tau_{s}}{b_{s}+b_{b}}\right)^{2}=\left(b_{b}-\tau_{s}\right) \frac{\tau_{s}\left(2 b_{b}^{2}+b_{s}^{2}+2 b_{b} b_{s}\right)-b_{b} b_{s}^{2}}{\left(b_{b}+b_{s}\right)^{2}}>0 .
\end{aligned}
$$

But for $b_{s}>b_{b}$, we have

$$
\begin{aligned}
\tau_{s}^{\min } & =\frac{b_{b}^{2}+4 b_{b} b_{s}+b_{s}^{2}}{2\left(b_{b}+2 b_{s}\right)}>b_{b}>\frac{b_{b} b_{s}^{2}}{2 b_{b}^{2}+b_{s}^{2}+2 b_{b} b_{s}} . \\
\text { So, } \tau_{s} & >\tau_{s}^{\min } \Rightarrow \tau_{s}>b_{b} \text { and } \tau_{s}>\frac{b_{b} b_{s}^{2}}{2 b_{b}^{2}+b_{s}^{2}+2 b_{b} b_{s}} \Rightarrow h_{s}^{s}>h_{s}^{b}
\end{aligned}
$$

It follows that $\tau_{s}>\tau_{s}^{\min }$ and $h_{s}<h_{s}^{b}$ imply that $h_{s}<h_{s}^{s}$ and sellers prefer multihoming, a contradiction. (2) We now show that $b_{s}>b_{b}, \tau_{s}>\tau_{s}^{\min }$, and $h_{s}^{\min }<h_{s}<h_{s}^{b}$ also imply that platforms prefer the competitive bottleneck environment. By contradiction, suppose that platforms prefer the two-sided-singlehoming environment. From Lemma 2, we know we need $K \equiv 8 \tau_{s}^{2}-8\left(b_{b}+b_{s}\right) \tau_{s}+\left(b_{b}^{2}+6 b_{b} b_{s}+b_{s}^{2}\right)>0$ and $h_{s}^{\min }<h_{s}<h_{s}^{\Pi}$. For $b_{s}>b_{b}$, we have both $K>0$ and $h_{s}^{\Pi}>h_{s}^{\min }$ if and only if $\tau_{s}<\frac{1}{2}\left(b_{b}+b_{s}\right)-\frac{1}{4} \sqrt{2}\left(b_{s}-b_{b}\right) \equiv \tau_{s}^{\max }$. Yet, it is easily shown that $b_{s}>b_{b}$ implies that $\tau_{s}^{\max }<\tau_{s}^{\min }$. It follows that for $b_{s}>b_{b}$ and $\tau_{s}>\tau_{s}^{\min }$, the inequality $h_{s}^{\Pi}<h_{s}^{\min }$ must hold and, therefore, that platforms prefer to allow sellers to multihome. 


\section{References}

[1] Anderson, S. and M. Peitz (2017). Media See-saws: Winners and Losers in Platform Markets. CEPR Discussion Paper 12214.

[2] Armstrong, M. (2006). Competition in Two-sided Markets. Rand Journal of Economics 37, 668-691.

[3] Armstrong, M. and J. Wright (2007). Two-sided Markets, Competitive Bottlenecks and Exclusive Contracts. Economic Theory 32, 353-380.

[4] Belleflamme, P. and M. Peitz (2015). Industrial Organization: Markets and Strategies. 2nd edition. Cambridge: Cambridge University Press.

[5] BKartA (2016). Market Power of Platforms and Networks. Working Paper B6-113/15.

[6] Evans, D. and R. Schmalensee (2012). The Antitrust Analysis of Multi-Sided Platforms. University of Chicago, Institute for Law and Economics Working Paper No. 623.

[7] Evans, D., A. Hagiu, and R. Schmalensee (2006). Invisible Engines: How Software Platforms Drive Innovation and Transform Industries, Cambridge, MA: MIT Press.

[8] OECD (2009). Two-Sided Markets. DAF/COMP(2009)20. December 17, 2009.

[9] Rochet, J.-C. and J. Tirole (2003). Platform Competition in Two-sided Markets, Journal of the European Economic Association 1, 990-1024.

[10] Rochet, J.-C. and J. Tirole (2006). Two-sided Markets: A Progress Report. Rand Journal of Economics 37, 645-667. 